\title{
The microbiome and mental health: Hope or hype?
}

\author{
Valerie $\mathrm{H}$. Taylor, MD, PhD
}

The biggest predictor of future behaviour is past behaviour, but in science we at times seem remarkably unable to learn from past experiences. Take the furor regarding the gut microbiome, especially as it pertains to mental illness. Less than 10 years ago the concept of microbial manipulation to treat mental illness was primarily hypothetical, yet we now have clinical trials underway to look the viability of fecal microbiota transplantation (FMT) as a treatment for major mental illness. While it is essential that the compelling preclinical work supporting the microbiome gutbrain axis ${ }^{1}$ be translated in a clinical population, and indeed that is a ubiquitous recommendation of many preclinical animal studies, it is essential to reflect on just how novel and revolutionary the concept of the gut-brain link really is. We also need to identify lessons to be learned from the past to ensure we recommend science and not science fiction going forward.

Prior to this recent renaissance, the concept of the gutbrain connection was prominent in the early 20th century when terms such as "autointoxication" and "intestinal toxemia" described a process whereby intestinally derived toxins were purported to influence health - often mental health specifically — via internal "poisoning" from the toxic contents of the colon. ${ }^{2}$ A paper published in The Journal of the American Medical Association in 1898, for example, suggested that microbial growth in the intestines associated with conditions such as a lack of stomach acid, could, under normal circumstances, be addressed via normal body processes, but the concern was that these detoxification pathways might be overrun in melancholia. ${ }^{3}$ This concept of vulnerability toward mental illness creating an environment where relatively normal amounts of stress suddenly result in illness is in keeping with the concept of allostatic load, ${ }^{4}$ but lacking sophisticated ways to understand the stress diathesis model and the role of neurotransmitters and inflammation, treatment focused on the identified problem area: the gut. This not only led to treatments targeted toward changing the gut via oral consumption of products comprising things such as lactic acid-producing bacteria, ${ }^{5}$ but also involved extreme measures such as gastrointestinal surgery for schizophrenia - a procedure associated with high mortality and little else. ${ }^{6}$

The flawed translation from scientific theory to clinical utility marked the beginning of the end for this school of thought and should be reflected on as the field, buoyed by strong preclinical work, again attempts to transition into the realm of treatment. One reason for the initial departure from the concept of autointoxication was purported to have been that, in parallel to the legitimate scientific interest in the effects of intestinal bacteria on health, charlatans were alert to the financial possibilities offered by the idea that cleaning out the colon could instantly improve well-being and began to sell a variety of alternative health products linked to improving your gut microflora and your sense of well-being. The irony should not be lost, given that the current global wellness industry had a $\$ 4.2$ trillion market in $2018,{ }^{7}$ and class action lawsuits have been settled for claims that probiotic-enriched foods can "boost the body's defenses" and "enhance the immune system."

Thankfully, the concept of gastrointestinal surgery to treat mental illness is no longer accepted, but the new methods being used to manipulate the gut microflora are not without risk. The primary tenet of FMT is that dysbiosis within the human host gut microbiome predisposes an individual to disease. The exact mechanisms through which this occurs have not yet been established, but several potential direct and indirect pathways exist through which the gut microbiota can modulate the gut-brain axis. These pathways include endocrine (cortisol), immune (cytokines) and neural (vagus and enteric nervous system) pathways, ${ }^{9}$ and the assumption is that introducing microflora from a healthy individual will help recolonize the system with a microbial pattern more in keeping with wellness either by establishing the new healthy microbiota or by allowing the host to "reset" their own microflora to a pre-illness state. As of yet, there is no clearly defined gold standard profile associated with euthymia, and factors such as diet, smoking and age can affect the microbiome. There is, however, work indicating that certain bacteria

Correspondence to: V.H. Taylor, Department of Psychiatry, Hotchkiss Brain Institute, University of Calgary, 3330 Hospital Dr NW, Calgary, AB T2N 4N1; valerie.taylor3@albertahealthservices.ca

DOI: $10.1503 / j p n .190110$ 


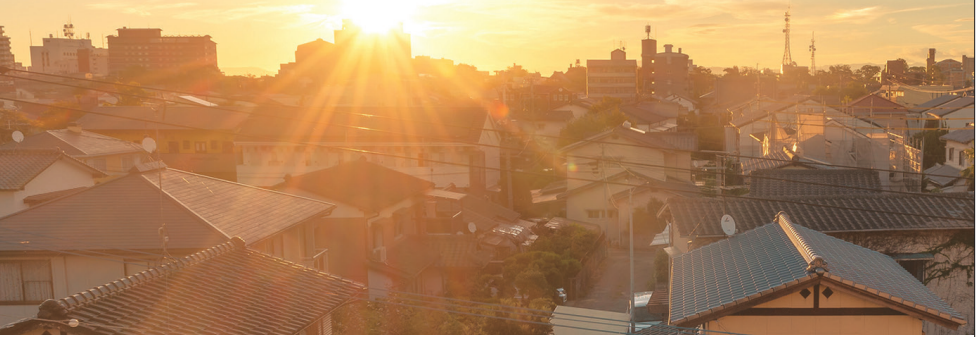

Please consult the product monograph at http://purdue.ca/ en/products/Foquest-PM for important information relating to:

- Contraindications in persons with known hypersensitivity or idiosyncrasy to sympathomimetic amines;

thyrotoxicosis; advanced arteriosclerosis; symptomatic cardiovascular disease; moderate to severe hypertension; glaucoma; history of drug abuse; during or within 14 days following administration of monoamine oxidase inhibitors

- The most serious warning and precaution regarding drug dependence/tolerance

- Other relevant warnings and precautions regarding: long-term effects of methylphenidate; use in patients who: are involved in strenuous exercise or activities, use other stimulants, or have a history of sudden/cardiac death; risk of sudden death, stroke and myocardial infarction; use of CNS stimulants in patients with cardiovascular or cerebrovascular conditions; use in patients with hypertension, taking alcohol; increase in seizure frequency, onset or exacerbation of motor or verbal tics; impairment in ability to operate machinery or vehicles; visual disturbances; psychiatric effects, including not for treatment of depression, or for use in treatment or prevention of normal fatigue states; exacerbation of psychosis symptoms in patients with pre-existing psychotic disorder; need to screen for risk of bipolar disorder in patients with comorbid depressive symptoms; need to monitor patients for: signs of suiciderelated behaviour; new psychotic or manic episodes, aggressive behaviour, marked anxiety, or agitation; caution regarding concomitant use of methylphenidate and serotonergic drugs (not recommended); precautions regarding priapism, peripheral vasculopathy, including Raynaud's phenomenon; use in pregnancy or breastfeeding; periodic laboratory testing during prolonged therapy; potential for misuse and dependence

- Conditions of clinical use, adverse reactions, drug interactions, and dosing information which have not been discussed in this piece.

The product monograph is also available by calling us at 1-800-387-5349.

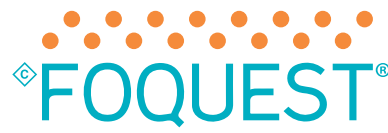

METHYLPHENIDATE HYDROCHLORIDE CONTROLLED RELEASE CAPSULES

REFERENCE: 1. ' ${ }^{C}$ FOQUEST ${ }^{\circledast}$ Product Monograph, Purdue Pharma, March 1, 2019.
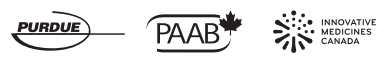

Purdue Pharma Inc
General Partner of

commandité de

Purdue Pharma

Pickering, Ontario LIW 3W8

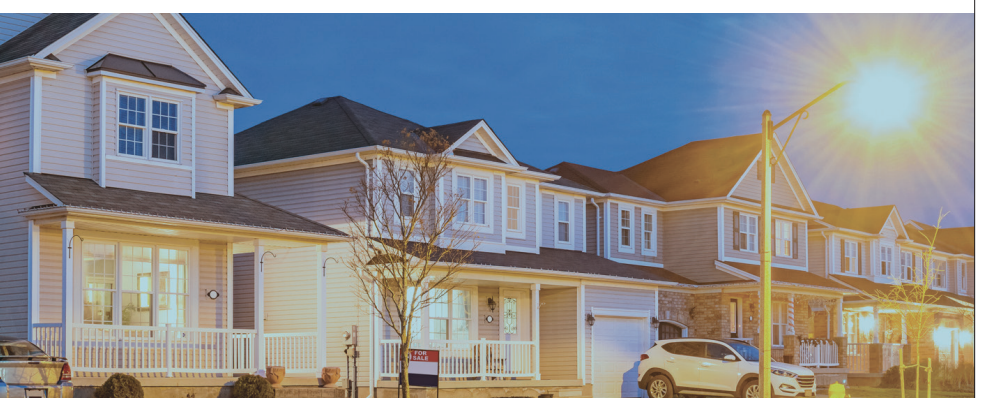

and microbial profiles are associated with illnesses such as depression. Naseribafrouei and colleagues reported that different members of the Bacteroidetes phylum are either positively or negatively correlated with depression; ${ }^{10}$ Jiang and colleagues found that Bacteroidetes, Proteobacteria and Actinobacteria are increased and Firmicutes decreased in depression; ${ }^{11}$ and Zheng and colleagues found that the relative abundance of Actinobacteria was increased, whereas that of Bacteroidetes was decreased, in depression compared with healthy controls. ${ }^{12}$ Using a module-based analytical framework, scientists have also recently assembled a catalogue of the neuroactive potential of sequenced gut prokaryotes and identified the microbial synthesis potential of the dopamine metabolite 3,4-Dihydroxyphenylacetic acid as correlating positively with mental quality of life, helping to initiate a more nuanced exploration of the exact mechanisms underlying the gut-brain connection. ${ }^{13}$

If we adopt the premise that changes in gut microflora subsequently affect behaviour via one of the above mechanisms, however, then the caveat is also true: all the illnesses purported to be amenable to remediation by therapies such as FMT can also be introduced via the same process. This issue was raised in an intriguing study in which germ-free mice were colonized with the fecal microbiota from healthy control individuals or patients with irritable bowel syndrome (IBS) with or without anxiety. The mice that received the IBS fecal microbiota, but not the healthy control fecal microbiota, exhibited faster gastrointestinal transit, intestinal barrier dysfunction, innate immune activation, and anxiety-like behaviour. As a consequence, the study authors noted that the results raised an important health issue related to fecal transplantation, and they strongly suggest that fecal transplant donors be screened for functional gut disorders and other conditions. ${ }^{14}$ This concept is further supported by work that showed that fecal microbiota transplantation of germ-free mice with "depression microbiota" derived from patients with depression resulted in depression-like behaviours compared with colonization with "healthy microbiota" derived from healthy control individuals. ${ }^{12}$ As noted in many areas of mental illness research, the causal association between the gut microbiome and mental illness is likely bidirectional and involves microbiome patterns that confer vulnerability toward illness and external stresses that create environments more prone to exacerbate such a biological vulnerability. What seems to be most important as the field moves forward, however, is the creation of new targets for treatment.

We need to manage expectations with respect to the therapeutic potential of microbial manipulation, and although there appears to be significant opportunity afforded by this area of research and much yet to be discovered, 2 recent meta-analyses indicate that probiotic supplementation has an overall insignificant effect on both mood symptoms ${ }^{15}$ and the symptoms of schizophrenia. ${ }^{16}$ In contrast, another systematic review and meta-analysis looking at pre- and probiotics for depression and anxiety found no difference over placebo for the prebiotic but a small but significant effect for the probiotic. ${ }^{17}$ This review included mainly studies of nonclinical 
community-based samples, however, which can be interpreted as representing work focused on participants who at baseline were too well to show meaningful impact. The results, therefore, can be used to endorse either viewpoint regarding the therapeutic potential of these compounds in the treatment of mental illness - which is not helpful.

As is true in many areas of mental health research, our diagnostic categorizations may be part of the issue, given that using DSM criteria for depression produces more than 200 different symptom profiles, all of which fit the major depressive disorder (MDD) diagnostic category. It may be, then, that probiotics are helpful for some, but not all, types of MDD. This area of research has the additional challenge of not only needing to identify what type of depression would be amenable to changes in the gut microflora, but also what type of probiotic should be used as an intervention and what the timing of the treatment should be. Results from a germ-free mouse study showed that central nervous system neurotransmission can be profoundly disturbed by the absence of a normal gut microbiota and that this aberrant neurochemical profile is resistant to restoration of a normal gut flora in later life. ${ }^{18}$ We also have systematic reviews of studies on the fecal microbiota in anorexia nervosa, the goal being to facilitate weight gain, ${ }^{19}$ and a review of the microbiome in the management of obesity, the goal being to facilitate weight loss, ${ }^{20}$ which speaks to our need to better understand what the science is and how we are going to translate this knowledge.

There is still a lack of understanding of what we are trying to change with treatment, as there is no microbial profile clearly associated with wellness, or vice versa, from a behavioural perspective. There is growing capacity, however, to move beyond causal associations and to begin to understand from a more mechanistic perspective exactly what the microbiome looks like when linked to wellness and to begin to think of the microbiome as perhaps contributing to the armamentarium of potential biomarkers we have available to help guide treatment. ${ }^{21}$ A recent large Dutch study has begun work at a population level to help create a dedicated reference database that will allow for a more sophisticated study of microbial neuroactive potential and provides population-scale evidence for microbiome links to mental health, while also acknowledging the issue of confounders. ${ }^{13}$ While provocative, the study also illustrates the need for multicentre work in this area, using validated instruments and solid metagenomic techniques to attempt to apply the cardiology model of thousands and tens of thousands of data points from large samples to impart rigour. If this is to be a game changer for mental illness, we need to change our approach. Otherwise we will, again, fail to truly know if a strong biological plausibility holds scientific credibility.

Affiliations: From the Department of Psychiatry, Hotchkiss Brain Institute, University of Calgary, Calgary, Alta., Canada.

Competing interests: V.H. Taylor has grant funding from the Stanley Medical Research Institute and the Weston Family Microbiome Initiative for microbiome research.

\section{References}

1. Dinan TG, Cryan JF. Brain-Gut-Microbiota Axis and Mental Health. Psychosom Med 2017;79:920-6.

2. Baron JH, Sonnenberg A. The wax and wane of intestinal autointoxication and visceroptosis-historical trends of real versus apparent new digestive diseases. Am J Gastroenterol 2002;97:2695-9.

3. Brower DR. Auto-intoxication in its relations to the diseases of the nervous system. JAMA 1898:575-7.

4. McEwen BS. Allostasis and the epigenetics of brain and body health over the life course: the brain on stress. JAMA Psychiatry 2017;74:551-2.

5. Metchnikoff E, Mitchell PC. The nature of man: studies in optimistic philosophy. New York and London: G. P. Putnam's sons; 1908.

6. Davidson J. Bayard Holmes (1852-1924) and Henry Cotton (18691933): surgeon-psychiatrists and their tragic quest to cure schizophrenia. J Med Biogr 2016;24:550-9.

7. Global Wellness Institute. Wellness now a $\$ 4.2$ trillion global industry. Available: https://globalwellnessinstitute.org/press-room/ press-releases/wellness-now-a-4-2-trillion-global-industry / (accessed 2019 May 8).

8. Danone agrees to pay $\$ 1.7 \mathrm{M}$ in yogurt health claims case. $C B C$ News 2012 Sept. 25. Available: https://www.cbc.ca/news/canada /montreal/danone-agrees-to-pay-1-7m-in-yogurt-health-claims -case-1.1206623 (accessed 2019 May 8).

9. Cryan JF, Dinan TG. Mind-altering microorganisms: the impact of the gut microbiota on brain and behaviour. Nat Rev Neurosci 2012;13:701-12.

10. Naseribafrouei A, Hestad K, Avershina E, et al. Correlation between the human fecal microbiota and depression. Neurogastroenterol Motil 2014;26:1155-62.

11. Jiang $\mathrm{H}$, Ling $\mathrm{Z}$, Zhang $\mathrm{Y}$, et al. Altered fecal microbiota composition in patients with major depressive disorder. Brain Behav Immun 2015;48:186-94.

12. Zheng $\mathrm{P}$, Zeng B, Zhou C, et al. Gut microbiome remodeling induces depressive-like behaviors through a pathway mediated by the host's metabolism. Mol Psychiatry 2016;21:786-96.

13. Valles-Colomer M, Falony G, Darzi Y, et al. The neuroactive potential of the human gut microbiota in quality of life and depression. New Microbiol 2019;4:623-32.

14. De Palma G, Lynch MD, Lu J, et al. Transplantation of fecal microbiota from patients with irritable bowel syndrome alters gut function and behavior in recipient mice. Sci Transl Med 2017;9:pii: eaaf6397.

15. Ng QX, Peters C, Ho CYX, et al. A meta-analysis of the use of probiotics to alleviate depressive symptoms. J Affect Disord 2018;228:13-9.

16. $\mathrm{Ng}$ QX, Soh AYS, Venkatanarayanan $\mathrm{N}$, et al. A systematic review of the effect of probiotic supplementation on schizophrenia symptoms. Neuropsychobiology 2019;78:1-6.

17. Liu RT, Walsh RFL, Sheehan AE. Prebiotics and probiotics for depression and anxiety: a systematic review and meta-analysis of controlled clinical trials. Neurosci Biobehav Rev 2019;102:13-23.

18. Clarke G, Grenham S, Scully P, et al. The microbiome-gut-brain axis during early life regulates the hippocampal serotonergic system in a sex-dependent manner. Mol Psychiatry 2013;18:666-73.

19. Schwensen HF, Kan C, Treasure J, et al. A systematic review of studies on the faecal microbiota in anorexia nervosa: future research may need to include microbiota from the small intestine. Eat Weight Disord 2018;23:399-418.

20. Marotz CA, Zarrinpar A. Treating obesity and metabolic syndrome with fecal microbiota transplantation. Yale J Biol Med 2016;89:383-8.

21. Lozupone M, La Montagna M, D'Urso F, et al. The role of biomarkers in psychiatry. Adv Exp Med Biol 2019;1118:135-62. 